Check for updates

Cite this: RSC Adv., 2018, 8, 14646

\title{
In vitro and in vivo assessment of nanostructured porous biphasic calcium phosphate ceramics for promoting osteogenesis in an osteoporotic environment $\dagger$
}

\author{
Kun Zhang, ${ }^{a}$ Jieyu Zhang, ${ }^{a}$ Kelei Chen, ${ }^{b}$ Xuefeng Hu, D *a Yunbing Wang, ${ }^{a}$ \\ Xiao Yang, ${ }^{\star a}$ Xingdong Zhang ${ }^{a}$ and Yujiang Fan (iD a
}

Treatment of bone defects in osteoporotic patients with bone substitutes is difficult, due to insufficient osseointegration. The development of appropriate biomaterials to solve the problem requires the assessment of the material performance in an osteoporotic environment, which is rarely investigated. Herein, nanostructured biphasic calcium phosphate (nBCP) ceramics were prepared via the incorporation of hydroxyapatite nanoparticles (HANPs) into porous biphasic CaP (BCP) substrates, leading to an increase of over $500 \%$ in the specific surface area. Primary osteoblasts harvested from osteoporotic rats were cultured on the nBCP ceramics, and it was found that the osteoblast functions, including proliferation, alkaline phosphatase activity, osteocalcin secretion and expression of osteogenic genes, were significantly enhanced compared with osteoblasts grown on non-nanostructured BCP ceramics. To further assess the osteoinduction ability, the ceramics were implanted in the femur of osteoporotic rats. Compared to the rats implanted with non-nanostructured BCP ceramics, a higher amount of mechanically matured bone was newly formed in the rats with nBCP ceramics after 6 weeks of implantation. Such enhanced osteoinduction ability of the $\mathrm{nBCP}$ ceramics may be due to the incorporated HANPs, as well as the nanostructured topography induced by the HANPs. These results indicate good in vitro and in vivo osteoinductivity of the $\mathrm{nBCP}$ ceramics in an osteoporotic environment and offer potential benefits for treating bone defects in osteoporotic patients.

\author{
Received 25th January 2018 \\ Accepted 2nd April 2018 \\ DOI: $10.1039 / \mathrm{c} 8 \mathrm{ra00768c}$ \\ rsc.li/rsc-advances
}

\section{Introduction}

Osteoporosis is characterized by deteriorating bone mass and microstructure due to disrupted dynamic balance between bone resorption and formation, and it has become a serious threat to public health. ${ }^{1}$ Even in developed regions such as North America and Europe, more than $30 \%$ of women and $20 \%$ of men are affected by osteoporosis and are at high risk of suffering bone fracture. ${ }^{2}$ Treatment of bone defects or fractures in osteoporotic patients is particularly difficult due to the impaired self-healing ability of bone. The current gold standard treatment is autografting, but this method has significant disadvantages such as limited supply and associated trauma after the harvesting surgery. Application of artificial bone substitutes could be a promising alternative. ${ }^{3}$ Nevertheless, after a bone

${ }^{a}$ National Engineering Research Center for Biomaterials, Sichuan University, 29 Wangjiang Road, Chengdu, 610064, China

${ }^{b}$ Zenmindes Biotech Co. Ltd, B2, CAS, No 9 4th Section of South Renmin Road, Chengdu, China. E-mail: huxuefeng@scu.edu.cn; xiaoyang114@scu.edu.cn; Fax: +86-28-85410246; Tel: +86-28-85417654

$\dagger$ Electronic supplementary information (ESI) available. See DOI: $10.1039 / \mathrm{c} 8 \mathrm{ra00768c}$ substitute is implanted in an osteoporotic patient, the osseointegration of the substitute is usually insufficient, significantly delaying the healing and adversely affecting the clinical outcome. ${ }^{4}$ Thus, development of artificial bone substitutes with good osteoinductivity in an osteoporotic environment is urgently needed.

Calcium phosphate (CaP) ceramics are widely used for artificial bone substitutes, since their chemical composition is similar to that of natural bone, and their osteoconductivity and osteoinductivity have been reported in previous in vitro and in vivo investigations. ${ }^{5-7}$ Beyond that, the porous structures of CaP ceramics may also contribute to the osteoconductivity and osteoinductivity. Interconnected macro-sized pores with sizes greater than $100 \mu \mathrm{m}$ allow for the ingrowth of newly formed bone, while micro-sized pores $(0.1-100 \mu \mathrm{m})$ are beneficial for the transport of nutrients and metabolized products. Aside from macro- and micro-sized pores, nano-sized pores $(<100 \mathrm{~nm})$ have recently been found to play positive roles in inducing osteogenesis..$^{7-9}$ However, the generation of nano-sized pores in $\mathrm{CaP}$ ceramics is not easy. The pores in CaP ceramics are commonly generated via foaming agents or porogens, but using these methods to generate nano-sized pores is difficult. 
During osteogenesis, mature osteoblasts secrete nano-sized hydroxyapatite (HA) particles onto Type I collagen (Col-1) fibers through matrix vesicles to initiate bone formation. ${ }^{\mathbf{1 0}}$ Inspired by this important role of HA nanoparticles (HANPs) in osteogenesis, researchers have started to investigate the possibility of incorporating synthetic HANPs in orthopedic implants to improve osseointegration, and have found that the regulation effects of synthetic HANPs on cells mainly depends on their dosage, size, shape and surface charge. ${ }^{\mathbf{1 1 - 1 3}}$ For example, highdose $\left(>500 \mu \mathrm{g} \mathrm{ml}^{-1}\right)$ and needle-shaped (aspect ratio $>2.5$ ) HANPs may induce cytotoxicity, while HANPs at a dosage of $<500 \mu \mathrm{g} \mathrm{ml}^{-1}$ and with a dot-like shape (aspect ratio $<1.5$ ) tend to enhance cellular attachment, proliferation and differentiation. ${ }^{\mathbf{1 4 , 1 5}}$ Moreover, HANPs may also exhibit cell-type-dependent regulation effects. HANPs can enhance the proliferation of osteoblasts but have no effect on normal hepatic and epithelial cells. ${ }^{16}$ Despite the fact that there are many interesting investigations into the application of HANPs in orthopedics, those studies were conducted using healthy cells or animals. In our previous study, it was found that osteoblasts harvested from osteoporotic rats responded differently to synthetic HANPs compared to those harvested from healthy rats. ${ }^{17}$ Thus, for medical devices used to treat bone defects of osteoporotic patients, it is necessary to assess their performance using in vitro and in vivo osteoporotic models.

In this study, HANPs were incorporated into porous $\mathrm{CaP}$ ceramics to investigate their potential to treat bone defects in osteoporotic patients. It was hypothesized that the incorporation of HANPs in porous CaP would induce the formation of nano-sized pores in the ceramics and enhance the osteoinductivity in an osteoporotic environment. To evaluate this hypothesis, the nanostructured ceramics were tested in in vitro and in vivo osteoporotic models.

\section{Materials and methods}

\section{Preparation of porous CaP ceramics}

The CaP precursor powders were synthesized using a wet precipitation method according to our previous study. ${ }^{\mathbf{1 8}}$ Briefly, $1000 \mathrm{ml}$ of $1.0 \mathrm{M} \mathrm{Ca}\left(\mathrm{NO}_{3}\right)_{2}$ was added dropwise into $600 \mathrm{ml}$ of $1.0 \mathrm{M}\left(\mathrm{NH}_{4}\right)_{2} \mathrm{HPO}_{4}$, and the $\mathrm{pH}$ was adjusted to 10.0 with $\mathrm{NH}_{4} \mathrm{OH}$. The slurry was kept at $80{ }^{\circ} \mathrm{C}$ for $24 \mathrm{~h}$, washed with deionized water and then dried with a SFDC-20 Spray Dryer (Shanghai Ohkawara Dryers Co., China), followed by breaking into powder with a jet mill.

The CaP precursor powder $(50 \mathrm{~g}), 10 \mathrm{ml}$ of $5 \%$ polyvinyl alcohol (PVA-1799, Chengdu Kelong Chemical Reagent Co., Ltd, China) aqueous solution, $10 \mathrm{ml}$ of $1 \%$ methyl cellulose (MC-400, Chengdu Kelong Chemical Reagent Co., Ltd, China) aqueous solution, and $30 \mathrm{ml}$ of $30 \mathrm{v} / \mathrm{v} \% \mathrm{H}_{2} \mathrm{O}_{2}$ solution were added into $10 \mathrm{ml}$ of deionized (DI) water. After stirring and sonication for $10 \mathrm{~min}$, the slurry was heated using a microwave oven for $2 \mathrm{~min}$. The foamed slurry was placed into customized cylindric molds and dried at $40{ }^{\circ} \mathrm{C}$ to obtain green bodies, which were subsequently sintered in a muffle furnace (LH15/14, Nabertherm $\mathrm{GmbH}$, Germany) at $1100{ }^{\circ} \mathrm{C}$ for $2 \mathrm{~h}$ to form porous $\mathrm{CaP}$ (cBCP) ceramics.

\section{Preparation of HANPs}

Thirty $\mathrm{ml}$ of $0.15 \mathrm{M}\left(\mathrm{NH}_{4}\right)_{2} \mathrm{HPO}_{4}$ solution was added dropwise into $30 \mathrm{ml}$ of $0.25 \mathrm{M} \mathrm{Ca}\left(\mathrm{NO}_{3}\right)_{2} \cdot 4 \mathrm{H}_{2} \mathrm{O}$ (Sigma-Aldrich, China) solution, and the $\mathrm{pH}$ was adjusted to $10.0 \mathrm{using} \mathrm{NH}_{4} \mathrm{OH}$. The solution was stirred for $2 \mathrm{~h}$ and then placed in an autoclave at $150{ }^{\circ} \mathrm{C}$ for $14 \mathrm{~h}$. The resultant precipitate was washed in DI water and anhydrous ethanol several times before freeze-drying for $24 \mathrm{~h}$, and then calcined in a furnace at $500{ }^{\circ} \mathrm{C}$ for $2 \mathrm{~h}$. The obtained HANPs were resuspended in DI water for subsequent use.

\section{Preparation of porous nanostructured BCP (nBCP) ceramics}

To obtain nBCP ceramics ( $\mathrm{nBCP} 1$ or $\mathrm{nBCP} 2$ ), the cBCP ceramics prepared previously were placed in 10 or $30 \mathrm{mg} \mathrm{ml}^{-1}$ HANP suspension under vacuum for $30 \mathrm{~min}$, respectively, followed by sonication for $15 \mathrm{~min}$. The vacuumization and sonication processes were repeated for another two rounds, and the cBCP ceramics were kept in the HANP suspension under vacuum for another $10 \mathrm{~h}$, to ensure permeation of the HANP suspension into the pores of the cBCP ceramics. After that, the obtained nBCP ceramics were dried at $60{ }^{\circ} \mathrm{C}$ followed by sintering in a muffle furnace at $650{ }^{\circ} \mathrm{C}$ for $8 \mathrm{~h}$.

\section{Ceramic characterization}

Size distribution of the HANPs was determined after $1 \mathrm{~h}$ of suspension in water using dynamic light scattering (DLS, Zetasizer Nano ZS Malvern, UK). The phase compositions and morphologies of the porous $\mathrm{CaP}$ ceramics were analyzed by $\mathrm{X}$ ray diffraction (XRD, X'Pert Pro, Philips, The Netherlands) and field emission scanning electron microscopy (FE-SEM, S4800, Hitachi, Japan), respectively. The porosity and specific surface area were measured with a surface area analyzer (GeminivII 2390t, Micromeritics), and the pore size distribution was obtained using mercury porosimetry (AutoPore IV 9500, Micromeritics).

\section{Culture of primary osteoblasts derived from osteoporotic rats}

All animal procedures were performed in accordance with the Guidelines for Care and Use of Laboratory Animals of Sichuan University and approved by the Animal Ethics Committee of Sichuan University. Mature female Sprague-Dawley rats (age: 3 months; body weight: $252.2 \pm 10.8 \mathrm{~g}$ ) were obtained from Laboratory Animal Center of Sichuan University (Chengdu, China), and acclimatized for 14 days under standard condition of temperature $\left(25^{\circ} \mathrm{C}\right)$ and a light-controlled environment $(12 \mathrm{~h}$ light/dark cycles). Osteoporotic rats were obtained 3 months after ovariectomy, and primary osteoblasts were isolated from the rats according to a previously established protocol from our group. ${ }^{19}$ Briefly, rats were euthanized by $\mathrm{CO}_{2}$ asphyxiation and tibia bones were harvested. The excised tibia was scraped thoroughly to remove soft tissues and then cut into small explants (1-2 $\mathrm{mm}$ in length). The bone explants were washed with antibiotic-antimycotic solution 5 times to remove bone marrow, and then placed in 6-well tissue culture plates. The explants were maintained in Dulbecco's modified Eagle's 
medium (DMEM, Gibco, USA) containing 10\% fetal bovine serum (FBS, Gibco, USA), 100 units per ml penicillin, $0.1 \mathrm{mg}$ $\mathrm{ml}^{-1}$ streptomycin and $100 \mu \mathrm{M}$ L-ascorbate (Sigma Aldrich, USA) in a humidified incubator at $37{ }^{\circ} \mathrm{C}$ under $5 \% \mathrm{CO}_{2}$ atmosphere, and the medium was changed every 3 days. Primary osteoblasts continuously exuded from the bone explants and attached to the culture plate, and the cells of second passage were used for in vitro tests.

\section{Thiazolyl blue tetrazolium bromide (MTT) and cellular morphology assays}

Primary osteoblasts were seeded on the porous CaP ceramics at a density of $10^{4}$ cells per $\mathrm{cm}^{2}$. On day 1,4 and 7 , the cells were incubated with $0.5 \mathrm{mg} \mathrm{ml} \mathrm{m}^{-1}$ MT solution in the medium for $4 \mathrm{~h}$, and the formed purple formazan salts were dissolved with dimethyl sulphoxide. The optical densities (OD) at the wavelength of $490 \mathrm{~nm}$ were recorded on a microplate reader (Varioskan Flash, Thermo Scientific, USA).

Cell morphology was observed with a confocal laser scanning microscope (CLSM, TCS SP5, Leica, German). The ceramics containing cells were washed with PBS after 4 days of culture, and the adhered cells were fixed in $4 \%$ paraformaldehyde for $30 \mathrm{~min}$. The fixed cells were then treated with $0.1 \%$ Triton X-100 in PBS for $10 \mathrm{~min}$ and the nonspecific binding sites were blocked with $1 \%$ BSA in PBS for $30 \mathrm{~min}$. The live cells were stained with fluorescein diacetate (Sigma, USA) in green and dead cells were stained with propidiumiodide (Sigma, USA) in red.

\section{Gene expression}

After 1, 4 and 7 days of culture, the total RNA of the cells was extracted using TRIzol reagent (Invitrogen, Carlsbad, USA) following the manufacturer's protocol. The concentration and purity of the extracted RNA were monitored using a Nanodrop 2000 UV spectrophotometer (ThermoScientific, USA). One microgram of the total RNA was reversely transcribed into complementary DNA (cDNA) using an iScriptTM cDNA Synthesis Kit (Bio-RAD, USA). Quantitative reverse transcriptase-polymerase chain reaction (qRT-PCR) was conducted using SsoFastTM EvaGreen Supermix (Bio-Rad, USA) on a CFX96TM real-time PCR system (CFX96, Bio-Rad, USA), and glyceraldehyde-3-phosphate dehydrogenase (GAPDH) was used as the housekeeping gene to calculate the expression values using the $2^{-\Delta \Delta \mathrm{Ct}}$ method.

\section{Alkaline phosphatase (ALP) activity and osteocalcin (OCN) secretion}

ALP activity and OCN secretion of the cells cultured on the ceramics were measured on day 4 and 7. Cells were lysed with RIPA lysis buffer (Cowin Biotech, Beijing, China) and centrifuged at $12000 \mathrm{~g}$ for $10 \mathrm{~min}$, and the cell lysate supernatant was collected for subsequent measurement. A pNPP Alkaline Phosphatase Assay Kit (Sensolyte, AnaSpec, Belgium) and BCA Protein Assay Kit (Pierce, IL, USA) were used, and the ALP activity was expressed as $\mu \mathrm{g} \mathrm{mg}^{-1}$ total protein. OCN levels were measured using an enzyme-linked immunosorbent assay
(ELISA) kit (SEA571Ra, Cloud-Clone Corp., China) according to the manufacturer's protocol.

\section{Implantation of ceramics in femoral defects of osteoporotic rats}

Sixteen osteoporotic rats were used for in vivo implantation of the ceramics into a femur metaphyseal defect. ${ }^{20}$ A dead-end bone defect ( $1.5 \mathrm{~mm}$ diameter and $3 \mathrm{~mm}$ depth) was created in the medial cortex of the right femur near the epiphyseal growth plate using a Kirschner wire, and the defect orientation was perpendicular to the sagittal axis of the femur (ESI Fig. S1 $\dagger$ ). Bone marrow was taken out and the defect was washed with saline. The ceramics were subsequently implanted in the defect and sealed with bone wax. Eight animals were used for each group, and none of the rats were excluded from the study, as there were no signs of distress or illness after the surgery. Four weeks after creating the bone defects, the animals were euthanized by $\mathrm{CO}_{2}$ asphyxiation, and femur samples were harvested, wrapped with gauze soaked in $0.9 \%$ saline and stored at $-20{ }^{\circ} \mathrm{C}$ until further analysis.

\section{Micro-computed tomography (micro-CT) analysis}

A pilot experiment of ex vivo X-ray imaging was performed on the surgery region of the rats to determine the optimal endpoint of the current study. Bone healing was observed for the rats from each group 15 days after the surgery (data not shown). Based on these preliminary results, 6 weeks was set as the endpoint herein, and a micro-CT imaging system ( $\mu \mathrm{CT} 80$, Scanco Medical AG, Bassersdorf, Switzerland) was used to evaluate the extent of osteogenesis within the defect. The harvested femur samples ( $n=8$ /group) were placed in a custommade holder to ensure that the long axis of the drilled channel was perpendicular to the axis of the X-ray beam. The resultant grayscale images had an isotropic voxel size of $25 \mu \mathrm{m}$ from cone-beam reconstruction $(70 \mathrm{kV}, 114 \mu \mathrm{A}, 700 \mathrm{~ms}$ integration time), and the global grayscale threshold embedded in the Scanco software was used for the analysis according to our previous work. $^{21,22}$ After the reconstruction, a $1 \times 1 \times 2 \mathrm{~mm}^{3}$ volume of interest was arranged centrally in the defect. For bone metaphysis analysis, the trabecular bone volume ratio (bone volume/total volume, $\mathrm{BV} / \mathrm{TV}$ ) and volumetric mean bone mineral density (BMD) were obtained using Scanco software according to the segmentation and threshold protocol we established earlier. ${ }^{23}$ All the micro-CT scanning and analysis data were collected by an expert (see the acknowledgement) who was blinded to the groups.

\section{Statistical analysis}

Statistical analyses were carried out using the SPSS v16 software and the results are reported as mean \pm standard deviation. Oneway ANOVA tests were performed by time to determine significant differences between groups for each time point. Bonferroni corrections were used for all the comparisons. Statistical significance was accepted at $P<0.05$. 

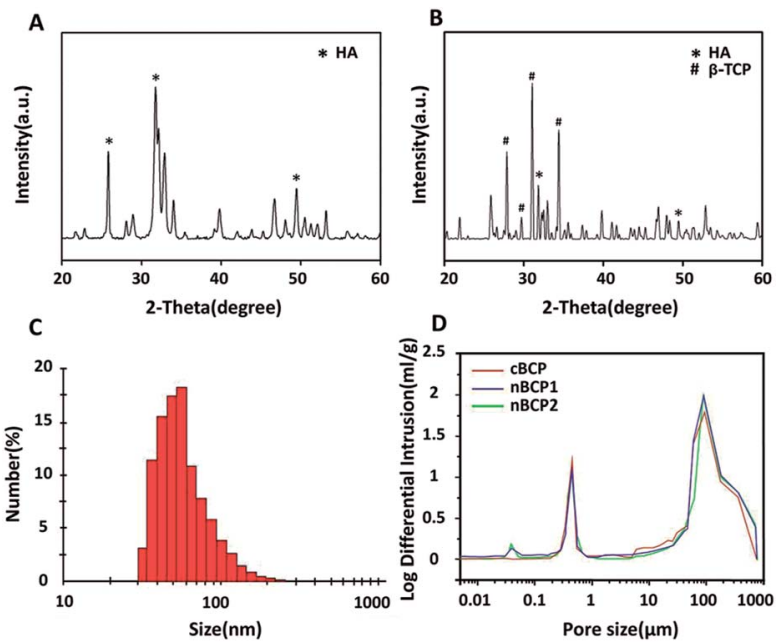

Fig. 1 The XRD spectra of (A) HANPs and (B) CBCP ceramics. * and \# indicate the characteristic signals of $H A$ and $\beta-T C P$, respectively. (C) Particle size distribution of HANPs. (D) Pore size distribution of the porous CBCP ceramics.

\section{Results}

\section{Phase composition and pore analysis}

The XRD spectrum in Fig. 1A confirmed that HA was successfully synthesized. ${ }^{5}$ Both the characteristic signals of HA and $\beta$ TCP were observed in the XRD spectrum of the obtained CBCP ceramics (Fig. 1B), indicating that the cBCP ceramics had a BCP structure, and the ratio of HA to $\beta$-TCP was $70 \% / 30 \%$. The obtained HA particles had a median size of $48 \mathrm{~nm}$ (Fig. 1C). As analyzed by mercury intrusion tests, the prepared porous cBCP ceramics did not have nano-sized pores (i.e. pore size $<0.1 \mu \mathrm{m}$ ), but after the incorporation of HANPs, nano-sized pores were formed (Fig. 1D). Compared to that of the cBCP ceramics, the surface grain sizes of the nBCP1 (prepared with $10 \mathrm{mg} \mathrm{ml}^{-1}$ HANP suspension) and nBCP2 (prepared with $30 \mathrm{mg} \mathrm{ml} \mathrm{m}^{-1}$ HANP suspension) ceramics decreased by over 20 -fold, and the specific surface area increased by over $300 \%$ for the nBCP1 sample and $500 \%$ for the nBCP2 sample (Table 1).

\section{Morphology of HANPs and BCP ceramics}

The TEM image (Fig. 2) shows that the obtained HANPs were nano-sized and cuboid-like shaped (50 $\mathrm{nm} \times 25 \mathrm{~nm}$ ), and the results were in accordance with the DLS result (Fig. 1C). Microsized surface grains on the $\mathrm{cBCP}$ ceramics were observed. In the

Table 1 Porosity, surface grain size, and specific surface area of porous BCP ceramics ${ }^{a}$

\begin{tabular}{lccc}
\hline & & $\begin{array}{l}\text { Surface grain } \\
\text { size }(\mathrm{nm})\end{array}$ & $\begin{array}{l}\text { Specific surface } \\
\text { area }\left(\mathrm{m}^{2} \mathrm{~g}^{-1}\right)\end{array}$ \\
\hline cBCP & 75.99 & $1000-3000$ & $0.87 \pm 0.01$ \\
nBCP1 & 73.84 & $40-60$ & $3.81 \pm 0.01^{*}$ \\
nBCP2 & 75.12 & $40-60$ & $5.56 \pm 0.02^{*} \#$
\end{tabular}

$a *$ and \# indicate a significant difference $(P<0.05)$ compared with the cBCP and nBCP1 ceramics.
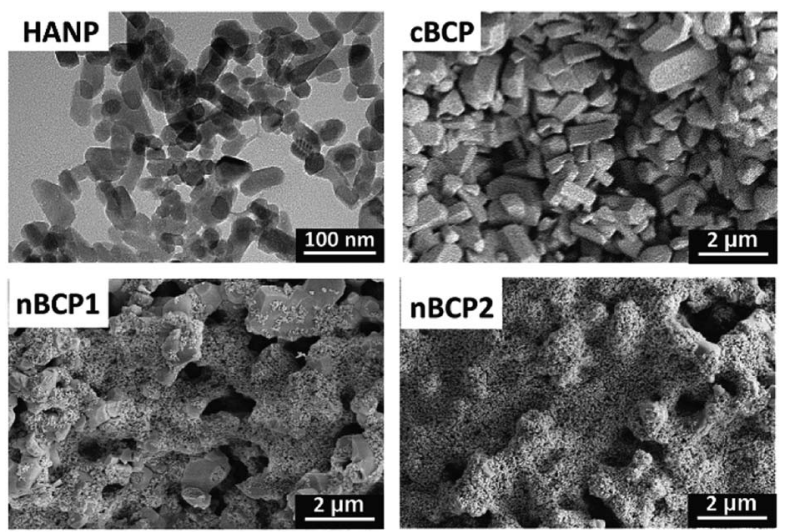

Fig. 2 TEM images of HANPs, and SEM images of $C B C P, n B C P 1$ and nBCP2 samples.

nBCP1 ceramics, HANPs were clearly seen on the surface of the BCP grains. The increase in the HANP concentration in the loading solution resulted in a higher amount of HANPs incorporated. When the loading concentration of the HANP suspension increased to $30 \mathrm{mg} \mathrm{ml}^{-1}$ (nBCP2), the surface micro-sized grains were fully covered with HANPs. Interestingly, although a large amount of HANPs were incorporated, the micro-sized and macro-sized pores in the cBCP1 and CBCP2 samples were not blocked, and the porosities of the $\mathrm{CBCP}$, nBCP1 and nBCP2 ceramics were similar (Table 1).

\section{Osteoblast proliferation and imaging}

Fig. 3A indicates that osteoblasts successfully migrated out from the bone explant harvested from osteoporotic rats. As shown in Fig. 3B, the harvested osteoporotic rat-derived osteoblasts were able to grow on the BCP ceramics over 7 days. On day 4 and 7 , the proliferation of the cells cultured on the nanostructured ceramics (nBCP1 and nBCP2) was promoted compared to those on the $\mathrm{CBCP}$ sample, but there was no significant difference between the proliferation results obtained for the nBCP1 and nBCP2 ceramics. The osteoblasts grown on the ceramics were also imaged using confocal microscopy after

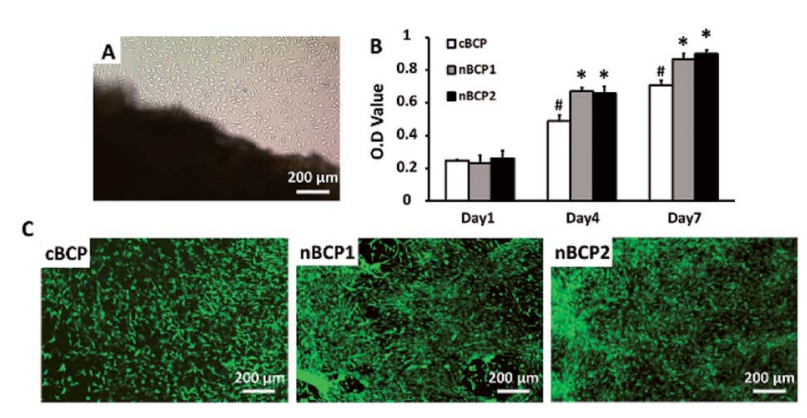

Fig. 3 (A) Establishment of osteoblast culture derived from bone explants of osteoporotic rats. (B) MTT assay of osteoblastic cells cultured on $\mathrm{CBCP}, \mathrm{nBCP} 1$ and $\mathrm{nBCP} 2$ samples over 7 days. * and \# indicate significant difference $(P<0.05)$ compared with the results of the $C B C P$ and $n B C P 1$ groups, respectively. (C) CLSM images of osteoblastic cells cultured on CBCP, nBCP1 and nBCP2 samples on day 7 . 

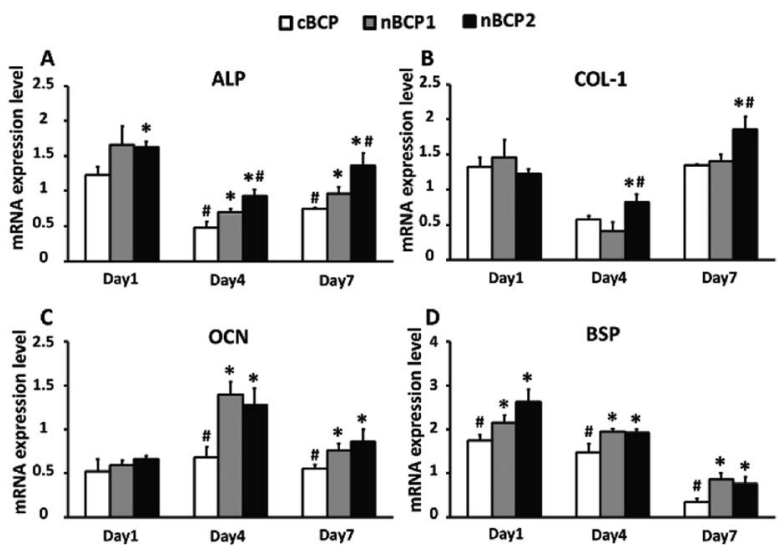

Fig. 4 (A) ALP, (B) COL-1, (C) OCN, and (D) BSP mRNA expression of osteoporotic rat-derived osteoblasts cultured on different BCP ceramics over 7 days. ${ }^{*}$ and \# indicate significant difference $(P<0.05)$ compared with the results of the $\mathrm{CBCP}$ and $\mathrm{nBCP} 1$ groups, respectively.

7 days of culture (Fig. 3C), and the results are consistent with those of the MTT assay.

\section{Osteoblast differentiation on the $\mathrm{BCP}$ ceramics}

The effect of the BCP ceramics on the differentiation of the osteoporotic rat-derived osteoblasts was assessed by osteoblastic gene expression, ALP activity and OCN secretion assays. As shown in Fig. 4, the nBCP1 ceramics enhanced the expression levels of three of the four chosen genes (ALP, OCN, and bone sialoprotein (BSP)) on day 4 and 7, while the nBCP2 ceramics upregulated the expression of ALP, Col-1, OCN and BSP genes. Compared to the cBCP ceramics, the nanostructured nBCP1 and nBCP2 ceramics enhanced ALP activity by $55 \%$ and $60 \%$ after 4 days of culture, respectively, while on day 7 , the corresponding increase was $72 \%$ and $154 \%$, respectively (Fig. 5). It is noted that after 7 days of culture, the ALP activity of osteoblasts on the nBCP2 ceramics was significantly higher than that on the nBCP1 ceramics.

As shown in Fig. 5, OCN secretion was also assessed, and in all the experimental groups, OCN secretion increased with prolonged culture period. On day 4, OCN secretion of osteoblasts cultured on the nBCP1 and nBCP2 ceramics was 105\% and $151 \%$ higher than that on the cBCP sample, while at the end of day 7 , the corresponding increase was $97 \%$ and $103 \%$, respectively. These results are consistent with the ALP activity
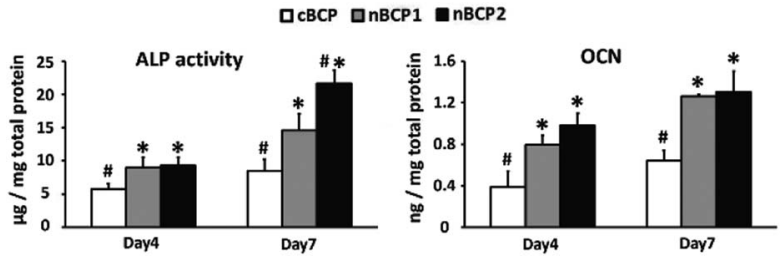

Fig. 5 ALP activity and OCN secretion for osteoporotic rat-derived osteoblasts cultured on different BCP ceramics over 7 days. * and \# indicate significant difference $(P<0.05)$ compared with the results of the $\mathrm{CBCP}$ and $\mathrm{nBCP1}$ groups, respectively.

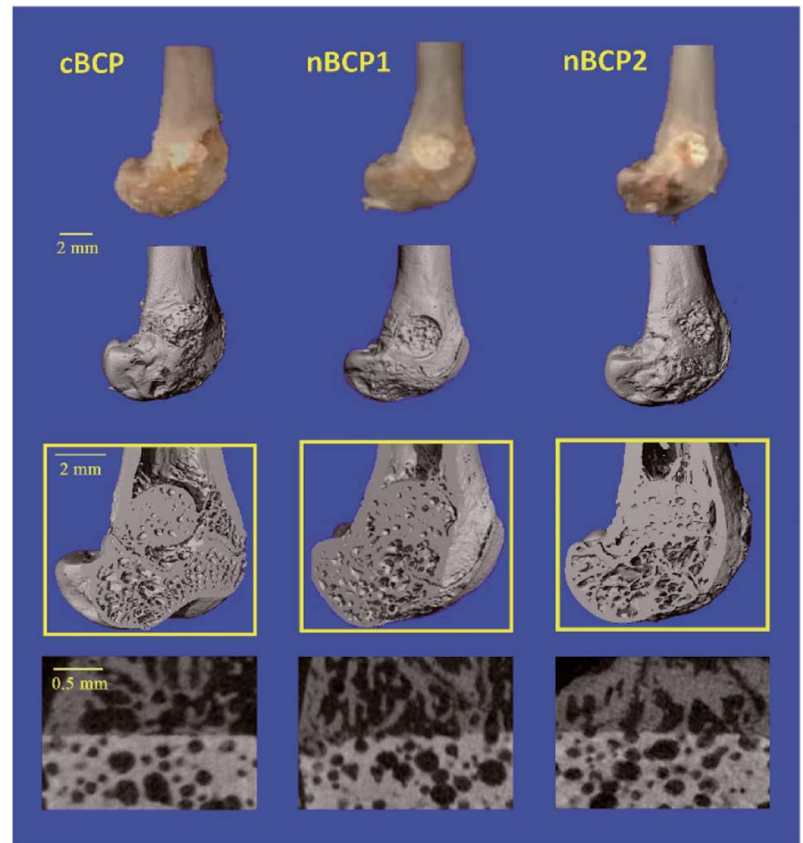

Fig. 6 Micro-CT images of the distal femurs 6 weeks after implantation with different BCP ceramics.

results, indicating that the nanostructured $\mathrm{BCP}$ ceramics are superior to the $\mathrm{CBCP}$ ceramics for enhancing osteoblast differentiation. Nevertheless, there was no significant difference in the promotion of osteoblast OCN secretion between the nBCP1 and $\mathrm{nBCP} 2$ ceramics.

\section{Micro-CT imaging and analysis of osteogenesis}

The contours of the recovering femoral metaphysis and the extracted cubical 3D images of the newly formed bone within the drilled defect are presented in the reconstructed micro-CT images (Fig. 6). The defect, where a nanostructured nBCP1 or nBCP2 ceramic was implanted, exhibited better recovery than that with cBCP ceramics. Compared to the cBCP group, a greater extent of osteogenesis, together with less intertrabeculae porosity, was observed for the nBCP1 or nBCP2 groups. The quantitative morphological indexes in the healing area obtained from the micro-CT results are summarized in Fig. 7. The nBCP2 group showed a 11\% higher BMD value than the $\mathrm{cBCP}$ group. Compared to the results of the cBCP group,
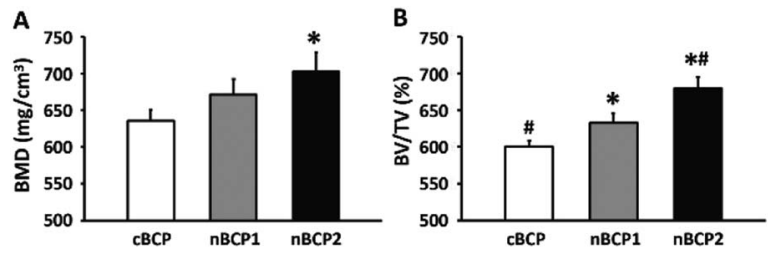

Fig. 7 (A) BMD value and (B) bone volume fraction (BV/TV) obtained from the micro-CT images 6 weeks after implantation with different BCP ceramics. * and \# indicate significant difference $(P<0.05)$ compared with the results of $\mathrm{CBCP}$ and $\mathrm{nBCP1}$ groups, respectively. 
$33 \%$ and $80 \%$ increases in the $\mathrm{BV} / \mathrm{TV}$ value were observed for the nBCP1 or nBCP2 groups, respectively.

\section{Discussion}

Porous CaP ceramics have been demonstrated to be excellent biomimetic scaffolds for bone tissue engineering, and both the porous structures and chemical properties are important for osteogenesis. ${ }^{24}$ Due to the different functions of macro-, microand nano-sized pores, many researchers have prepared various scaffolds with hierarchical pores which are promising for bone tissue engineering.. ${ }^{25-27}$ However, even though hierarchical pores were obtained in those studies, the pore size was $>100 \mathrm{~nm}$ and nano-sized pores were difficult to achieve. In this study, in addition to the formation of macro- and micro-sized pores (Fig. 1D), the incorporation of HANPs also led to nano-sized pores, resulting from the gaps among the incorporated HANPs in the nBCP1 and nBCP2 ceramics (Fig. 2). It was noted that the porosity of the $\mathrm{nBCP} 1$ and $\mathrm{nBCP} 2$ ceramics was similar to that of the сBCP ceramics (Table 1), indicating that the original macro- and micro-sized pores in the $\mathrm{CBCP}$ ceramics were not blocked after HANP incorporation. In addition to the hierarchical porous structures, there were also HANPs on the surfaces of the ceramics, which may also enhance the differentiation of osteoblasts based on the results of our previous investigation. ${ }^{17}$

In many previous investigations, commercial osteoblast-like cell lines (i.e., osteosarcoma cells) have been used as in vitro models to test the interactions between the biomaterials and bone cells. ${ }^{28-30}$ However, these models may not be suitable to reflect cellular responses in osteoporotic conditions because osteoblastic cells derived from healthy and osteoporotic rats exhibit different behavior towards the same stimuli. ${ }^{31}$ Our group has also reported that osteoblastic cells derived from osteoporotic rats using the most widely-used outgrowth protocol developed by Cornelissen's group ${ }^{32,33}$ exhibited significantly lower ALP activity. Furthermore, the matrix mineralization ability and expression levels of osteoblastic genes such as OCN, BSP and osterix were lower compared with those derived from healthy rats. ${ }^{17}$ The commonly-used protocols of osteoblast harvesting are enzymatic extraction of calvaria and bone marrow cultures. However, these methods were not suitable for the current study. Enzymatic extraction is not effective in digesting the adult bone in our 6 month old ovariectomized rats. For bone marrow culture, the harvested cells are a mixture of cells including general stromal cells, endothelial cells, and fibroblasts, and the cellular viability was too low. As indicated by Declercq et al., ${ }^{33,34}$ bone explant culture from adult long bone is more reliable than enzymatic extraction of fetal calvaria or bone marrow culture. Thus, in the present study, osteoblasts were harvested using a protocol created in a previous study of our group. ${ }^{19}$

Our results of the in vitro test showed that compared with the cBCP ceramics, the nanostructured $\mathrm{nBCP} 1$ and $\mathrm{nBCP} 2$ ceramics promoted the proliferation of osteoporotic rat-derived bone cells (Fig. 3). To test the effect of these nanostructured ceramics on osteoblastic differentiation of osteoporotic rat-derived bone cells, the expression levels of the four-bone matrix maturationrelated genes were evaluated. ALP and BSP genes are activated at an early stage of osteoblastic differentiation..$^{34}$ The OCN gene is responsible for calcium deposition and bone mineralization, and the Col-1 gene regulates the production of Col- 1 , which is the major organic component of bone..$^{35}$ Therefore, the significantly higher expression levels of the ALP, Col-1, OCN and BSP genes in the nBCP1 and nBCP2 groups compared with those of the cBCP group (Fig. 4) indicate that the nanostructured ceramics promoted osteogenic differentiation and mineralization of the osteoblasts derived from osteoporotic rats. These conclusions were further confirmed by the protein secretion results (ALP activity and OCN secretion in Fig. 5). Comparison of the results obtained with the nBCP1 and nBCP2 ceramics reveals that the nBCP2 ceramics are superior to the nBCP1 ceramics in promoting the functions of osteoporotic rat-derived bone cells, possibly due to its higher amount of incorporated HANPs.

Consistent with the in vitro results (Fig. 3-5), a higher amount of newly formed bone in the defect area was visualized in the rats implanted the nanostructured ceramics compared with those implanted with the cBCP ceramics (Fig. 6). The quantification results of BMD and BV/TV ratio also exhibited a similar trend (Fig. 7).

The observed osteoinductive effect of the nanostructured nBCP ceramics may result from the incorporated HANPs and the nanostructures formed by the HANPs. In our previous study, we found that HANPs can promote intracellular calcium levels in osteoporotic rate-derived bone cells, and thus enhance their osteoblastic differentiation. ${ }^{17}$ Such an increase in the intracellular calcium levels could be due to endocytosis of the HANPs in the cells ${ }^{36}$ or HANP-induced upregulated expression of sarcoplasmic/endoplasmic reticulum calcium ATPase2, leading to calcium refilling into the endoplasmic reticulum. ${ }^{37}$

Natural bone includes building blocks of hierarchical scales such as mesoscale calcified layers, micro-sized collagen fibers and nano-sized HA crystals, which regulate the functions of bone cells via biochemical and mechanical signals. There is growing evidence that nano-sized $\mathrm{CaP}$ is more similar to the HA crystals in natural bone, and it exhibits greater physicochemical and biological effects than conventional $\mathrm{CaP}$ materials in bone tissue engineering. ${ }^{38-40}$ Thus, the nanostructures formed in the nBCP ceramics may be another factor responsible for its higher osteoinductivity compared with the cBCP ceramics. The formed nano-sized pores increased the surface area of the material (Table 1), providing more binding sites for cellular receptors and enhancing the adsorption and retention of circulating osteogenic cytokines, which contribute to osteogenesis. ${ }^{41}$ In addition, the nano-sized pores may also induce changes in membrane receptors of the attached cells and activate the downstream transduction pathways for osteogenesis. ${ }^{42}$

\section{Conclusion}

Incorporation of HANPs into porous $\mathrm{cBCP}$ substrates led to the formation of nanostructures in the material, while maintaining 
the high porosity (75\%) of the BCP ceramics. The formed nanostructures increased the specific surface area to $5.56 \pm 0.02$ $\mathrm{m}^{2} \mathrm{~g}^{-1}$ (the value for the BCP ceramics was $0.87 \pm 0.01 \mathrm{~m}^{2} \mathrm{~g}^{-1}$ ). After 7 days of culture, the nBCP ceramics promoted the proliferation of primary osteoblasts harvested from osteoporotic rats by $25 \%$ compared with the $\mathrm{cBCP}$ ceramics. In addition to the effect on cellular proliferation, the nBCP ceramics also significantly enhanced ALP activity, OCN secretion and the expression of ALP, COL-1, OCN and BSP genes, clearly demonstrating an enhancement effect on osteoblast differentiation. The in vivo bone defect model generated in osteoporotic rats further proved that the nanostructured nBCP ceramics were better than the cBCP ceramics, which did not possess nanostructures for inducing osteogenesis. Thus, our findings indicate the good osteoinductivity of the $\mathrm{nBCP}$ ceramics in in vitro and in vivo osteoporotic environments, and pave the way for developing new strategies to treat bone defect in osteoporotic patients.

\section{Conflicts of interest}

The authors declare no competing financial interests.

\section{Acknowledgements}

This work was supported by National Key Research and Development Program of China (2017YFB0702600, 2017YFB0702603), the Fundamental Research Funds for the Central Universities (YJ201626), Sichuan University Research Fund for Full-time Postdoctoral (2018SCU12056) and National Natural Science Foundation of China (grant no. 31500774). The authors would like to thank Dr Li Chen from Analytical \& Testing Center of Sichuan University for her help with the micro-CT scanning and analysis.

\section{References}

1 T. D. Rachner, S. Khosla and L. C. Hofbauer, Lancet, 2011, 377, 1276-1287.

2 E. Hernlund, A. Svedbom, M. Ivergard, J. Compston, C. Cooper, J. Stenmark, E. V. McCloskey, B. Jonsson and J. A. Kanis, Arch. Osteoporos., 2013, 8, 136.

3 K. A. Egol, A. Nauth, M. Lee, H. C. Pape, J. T. Watson and J. Borrelli Jr, J. Orthop. Trauma, 2015, 29(suppl. 12), S10-S14.

4 J. M. Feron and R. Mauprivez, Injury, 2016, 47(suppl. 1), S10S14.

5 J. Wang, Y. Chen, X. Zhu, T. Yuan, Y. Tan, Y. Fan and X. Zhang, J. Biomed. Mater. Res., Part A, 2014, 102, 4234-4243.

6 Z. Tang, Y. Tan, Y. Ni, J. Wang, X. Zhu, Y. Fan, X. Chen, X. Yang and X. Zhang, Mater. Sci. Eng., C, 2017, 70, 10001010.

7 H. Yuan, H. Fernandes, P. Habibovic, J. de Boer, A. M. Barradas, A. de Ruiter, W. R. Walsh, C. A. van Blitterswijk and J. D. de Bruijn, Proc. Natl. Acad. Sci. U. S. A., 2010, 107, 13614-13619.
8 P. Habibovic, H. Yuan, C. M. van der Valk, G. Meijer, C. A. van Blitterswijk and K. de Groot, Biomaterials, 2005, 26, 3565-3575.

9 P. Habibovic, T. M. Sees, M. A. van den Doel, C. A. van Blitterswijk and K. de Groot, J. Biomed. Mater. Res., Part A, 2006, 77, 747-762.

10 H. Zhou and J. Lee, Acta Biomater., 2011, 7, 2769-2781.

11 L. Jiang, Y. Li, C. Xiong, S. Su and H. Ding, ACS Appl. Mater. Interfaces, 2017, 9, 4890-4897.

12 A. K. Teotia, D. B. Raina, C. Singh, N. Sinha, H. Isaksson, M. Tagil, L. Lidgren and A. Kumar, ACS Appl. Mater. Interfaces, 2017, 9, 6816-6828.

13 W. Nie, C. Peng, X. J. Zhou, L. Chen, W. Z. Wang, Y. Z. Zhang, P. X. Ma and C. L. He, Carbon, 2017, 116, 325-337.

14 J. L. Xu, K. A. Khor, J. J. Sui, J. H. Zhang and W. N. Chen, Biomaterials, 2009, 30, 5385-5391.

15 N. S. Remya, S. Syama, V. Gayathri, H. K. Varma and P. V. Mohanan, Colloids Surf., B, 2014, 117, 389-397.

16 Y. Sun, Y. Chen, X. Ma, Y. Yuan, C. Liu, J. Kohn and J. Qian, ACS Appl. Mater. Interfaces, 2016, 8, 25680-25690.

17 R. Zhao, P. Xie, K. Zhang, Z. Tang, X. Chen, X. Zhu, Y. Fan, X. Yang and X. Zhang, Acta Biomater., 2017, 59, 338-350.

18 X. D. Zhu, H. J. Zhang, H. S. Fan, W. Li and X. D. Zhang, Acta Biomater., 2010, 6, 1536-1541.

19 F. Qing, P. Xie, Y. S. Liem, Y. Chen, X. Chen, X. Zhu, Y. Fan, X. Yang and X. Zhang, J. Orthop. Res., 2016, 34, 1147-1157.

20 E. Tatsukawa, Y. Gonda, M. Kamitakahara, M. Matsuura, M. Ushijima, Y. Shibata, I. Yonezawa, M. Fujiwara, K. Ioku and T. Ikeda, J. Orthop. Res., 2014, 32, 189-196.

21 Z. Tang, Z. Y. Tan, Y. Ni, J. Wang, X. Zhu, Y. Fan, X. Chen, X. Yang and X. Zhang, Mater. Sci. Eng., C, 2017, 70, 10001010.

22 Y. Zhu, K. Zhang, R. Zhao, X. Ye, X. Chen, Z. Xiao and X. Yang, Biomaterials, 2017, 147, 133-144.

23 X. Yang, P. Muthukumaran, S. DasDe, S. H. Teoh, H. Choi, S. K. Lim and T. Lee, Bone, 2013, 52, 308-317.

24 S. Wu, X. Liu, K. W. K. Yeung, C. Liu and X. Yang, Mater. Sci. Eng., $C, 2014, \mathbf{8 0}, 1-36$.

25 M. Chen, D. Q. Le, A. Baatrup, J. V. Nygaard, S. Hein, L. Bjerre, M. Kassem, X. Zou and C. Bunger, Acta Biomater., 2011, 7, 2244-2255.

26 Y. Hu, J. Chen, T. Fan, Y. Zhang, Y. Zhao, X. Shi and Q. Zhang, Colloids Surf., B, 2017, 157, 93-100.

27 C. Wang, Q. Zhao and M. Wang, Biofabrication, 2017, 9, 025031.

28 B. Kim, R. Ventura and B. T. Lee, J. Tissue Eng. Regener. Med., 2017, 1-12.

29 A. J. Nathanael, A. Oyane, M. Nakamura, I. Sakamaki, E. Nishida, Y. Kanemoto and H. Miyaji, ACS Appl. Mater. Interfaces, 2017, 9, 22185-22194.

30 J. Zhang, L. Sun, X. Luo, D. Barbieri, J. D. de Bruijn, C. A. van Blitterswijk, L. Moroni and H. Yuan, J. Tissue Eng. Regener. Med., 2017, 11, 3273-3283.

31 K. S. Leung, C. Y. Li, Y. K. Tse, T. K. Choy, P. C. Leung, V. W. Hung, S. Y. Chan, A. H. Leung and W. H. Cheung, Osteoporosis Int., 2014, 25, 1785-1795. 
32 H. Declercq, N. Van den Vreken, E. De Maeyer, R. Verbeeck, E. Schacht, L. De Ridder and M. Cornelissen, Biomaterials, 2004, 25, 757-768.

33 H. A. Declercq, R. M. Verbeeck, L. I. De Ridder, E. H. Schacht and M. J. Cornelissen, Biomaterials, 2005, 26, 4964-4974.

34 S. W. Ha, H. L. Jang, K. T. Nam and G. R. Beck Jr, Biomaterials, 2015, 65, 32-42.

35 M. B. Lazebnik, M. I. Tussie-Luna, P. W. Hinds and A. L. Roy, J. Biol. Chem., 2009, 284, 36234-36239.

36 F. Qing, Z. Wang, Y. Hong, M. Liu, B. Guo, H. Luo and X. Zhang, J. Mater. Sci.: Mater. Med., 2012, 23, 2245-2251.

37 S. Patergnani, C. Giorgi, S. Maniero, S. Missiroli, P. Maniscalco, I. Bononi, F. Martini, G. Cavallesco, M. Tognon and P. Pinton, Oncotarget, 2015, 6, 23427-23444.
38 P. Wang, L. Zhao, J. Liu, M. D. Weir, X. Zhou and H. H. K. Xu, Bone Res., 2014, 2, 14017.

39 J. Zhang, W. Liu, V. Schnitzler, F. Tancret and J.-M. Bouler, Acta Biomater., 2014, 10, 1035-1049.

40 H. Wang, M. Bongio, K. Farbod, A. W. Nijhuis, J. van den Beucken, O. C. Boerman, J. C. van Hest, Y. Li, J. A. Jansen and S. C. Leeuwenburgh, Acta Biomater., 2014, 10, 508-519.

41 J. C. Fricain, S. Schlaubitz, C. Le Visage, I. Arnault, S. M. Derkaoui, R. Siadous, S. Catros, C. Lalande, R. Bareille, M. Renard, T. Fabre, S. Cornet, M. Durand, A. Leonard, N. Sahraoui, D. Letourneur and J. Amedee, Biomaterials, 2013, 34, 2947-2959.

42 H. Nikukar, S. Reid, P. M. Tsimbouri, M. O. Riehle, A. S. G. Curtis and M. J. Dalby, ACS Nano, 2013, 7, 2758-2767. 